\title{
The Effect of the Training of Gradual Muscular Relaxation in Reducing Psychological Stress Among Students of The Faculty of Sports Science and Physical Activity
}

\author{
Haggag $\mathbf{M}^{*}$ \\ Department of Sport Psychology, King Saud University, Saudi Arabia
}

*Corresponding author: Haggag M, Department of Sport Psychology and Biomechanics \& Motor Behavior, College of Sport Sciences \& Physical Activity, King Saud University, Saudi Arabia

\begin{abstract}
Background: Psychological stress is a major risk factor for the development and progression of a number of diseases, including cardiovascular disease, cancer, arthritis, and major depression. It greatly affects both older and younger ages globally. Thus, searching for new non-drug training strategies for ameliorating psychological stress are recommended.

Aim: the aim of the present study was to characterize the rate of psychological stress among students of faculty of sports sciences and to evaluate the efficacy of a particular relaxing gradual technique called progressive muscle relaxing (PMR) for ameliorating the mood and for returning the psychological stress parameters into normal levels.

Methods: Psychological stress was evaluated in 40 of students of faculty of sports sciences aged 18-21 years old. They are classified into two groups according to the scores of psychological stress; control group $(\mathrm{n}=20)$ and Psychological stress group $(n=20)$. For the treatment of psychological stress, both groups participated in PMR- training intervention ( 2 times /week) for 12 weeks.

Results: In this study, psychological stress parameters were significantly reported in 50\% ( 20/40) of the participated students. PMR- training interventions showed a gradual significant reduction in total psychological stress scores and other related parameters; family stress, study stress, economic stress, and social stress respectively. These results greatly support the use of PMR gradual interventions for the reduction of psychological stress among younger ages.

Conclusion: Application of PMR training interventions for 12 weeks gradually and significantly improve psychological stress among the students of faculty of sports sciences. It could be supported as non-drug training treatment among psychological stress holders, particularly in younger ages.
\end{abstract}

Keywords: Gradual Muscular Relaxation; Sports Psychology; Psychological Stress

\section{Introduction}

Psychological stress is a major risk factor for the development and progression of a number of diseases, including cardiovascular disease, cancer, arthritis, and major depression [1]. A fact known by the scientific community is that emotions in general, and stress in particular, produce interrelated functional changes, mainly through the vegetative nervous system, the endocrine system, and the immune system [1,2]. Subjects with a protracted state of tension greatly suffer from psychological, physiological and behavioral effects which overtime an lead to harmful consequences [2]. The contemporary society maintains individuals in a constant struggle for success without taking into account their needs and how much all this costs them. For professional sportsmen, mood and level of stress have been always considered aspect they must learn to control. Moreover, an increase of alteration of mood states and of levels of stress has reported in young students [3-5]. Also, it was reported that Youth is the period of contradictions in which 
the individuals experience many emotional, behavioral, sexual, economic, academic and social conflicts which result in social and physical changes [6-7].

Thus, in this period the emotional health of university youth becomes one of the important components of social health [6-8]. For this reason, it is important to detect the status of psychological stress among younger ages and to find good solutions to overcome these contradictions.

Progressive muscle relaxation (PMR) is a technique for reducing stress and anxiety by alternately tensing and relaxing the muscles as previously reported [9-11]. It mainly requires both a physical and a mental component. Previous research reports recommended several benefits of PMR technique including a reduction in salivary cortisol levels and generalized anxiety [10] decreased blood pressure and heart rate [10,12], decreased headaches [13], better management of cardiac rehabilitation [14] improvement of quality of life of patients with chronic diseases or following severe surgical operations [15-16]. Thus it is better to use PMR technique in for ameliorating psychological stress among younger ages. Thus, it is important to identify the psychological stress among younger ages and find out an alternative non-drug remedy to overcome this stress. In this regard, the aim of the present study was to characterize the rate of psychological stress among students of faculty of sports sciences and to evaluate the efficacy of a particular relaxing gradual technique called progressive muscle relaxing (PMR) for ameliorating the mood and for returning the psychological stress parameters into normal levels.

\section{Materials and Methods}

\section{Participants}

A total of 40 of Students of Faculty of Sport Sciences aged 18-21 years old from College of Sport Sciences \& Physical Activity, King Saud University, Riyadh, Saudi Arabia. were enrolled in this study. Students who had serious acute or chronic health problems such as diabetes, endocrine disorders, cardiovascular disease, systemic infections, musculoskeletal disorders, or concentration problems or psychological disorders such as depression, or post-traumatic stress disorders that might change the psychological stress parameters were excluded from this study. Based upon psychological stress score (PSS), the students classified into two groups; control $(n=20)$ and Psychology stress group $(n=20)$. At a statistical power of $96 \%$ and a significance level of $\leq 0.05$, the sample size of the study was estimated to be 42 participants to compensate for an estimated 12 $\%$ dropout rate. The study was approved by the Research Ethics Committee of King Saud University, and all participants gave signed informed consent. Baseline demographic and characteristics of all participants were shown in Table 1

\section{Assessments of Psychological Stress}

Pre-validated psychological stress scales were applied to evaluate the degree of social and psychological stress measures among students. These measures were evaluated according to previously performed stress scales and that test-retest reliability coefficient of 0.80 or higher for these statistics are indicative of acceptable test-retest reliability $[17,18]$.

\section{Assessments of Progressive Muscle Relaxation (PMR)}

Progressive muscle relaxation (PMR) is a technique for reducing stress and anxiety by alternately tensing and relaxing the muscles as previously reported $[9,10]$. In this techniques all students performed the PMR training interventions 2 times /week for 12 weeks. In this training program, the physical component involves tensing and relaxing of muscle groups over the legs, abdomen, chest, arms, and face. In a sequential pattern, with eyes closed, the tension of the individual place in a given muscle group purposefully for approximately 10 seconds and then releases it for 20 seconds before continuing with the next muscle group [9-11]. The mental component requires that the individual focuses on the distinction between the feelings of tension and relaxation. Relaxation must be attempted in order to reduce pain or pain perception and tension, create a pleasant mental state, enhance the performance of physical activities and help in the relationship with others [11-12].

\section{Statistical Analysis}

An SPSS software (Statistical Package for the Social Sciences, version 18.0, SPSS Inc. Chicago, IL, USA) was used to perform statistical analyses. The qualitative variables were presented in terms of frequencies and percentage, and the quantitative variables were presented using mean and standard deviation. For analyses within the groups, we used a t-test for paired data. The unpaired t-test was used for within and between groups. The comparison and correlation of the studied parameters were investigated using both Student's t-test and Pearson's correlation coefficient, respectively Values at $\mathrm{p}<0.05$ were considered statistically significant.

\section{Results}

In this study, all psychological stress parameters; family stress, study stress, economic stress, and social stress, and the effect of progressive muscle training interventions on the degree of psychological stress were characterized in 40 healthy university students. Based on the degree of the severity of psychological stress scores, the participants classified into two groups; control healthy $(n=20)$ and psychological stress $(n=20)$ as shown in (Table 1). There is no significant difference in age, BMI, but WHR of adiposity showed little non-significant change in psychological students compared with healthy controls (Table 1). The results showed that students with higher scores of psychological stress parameters require more non-drug support interventions.

In this current study, both control and psychological students participated in PMR training interventions for 12 weeks $(2$ times /week) and the effect of PMR -exercise on psychological stress was evaluated as shown in (Table 2) and (Figure 1). PMR-Post results showed that all psychological parameters were significantly declined and improved in all healthy $(\mathrm{P}=0.01)$ and students with psychological stress $(\mathrm{P}=0.001)$ as shown in (Table 2). The data showed that PMR training interventions program improve psychological stress gradually during the period of treatment as shown in (Figure 1). 
Table 1: Baseline demographic characteristics of the students of Faculty of Sport Sciences $(n=40)$.

\begin{tabular}{|c|c|c|c|}
\hline Variables & Control (n=20) & Psychology stressGroup (n=20) & P-value \\
\hline Age (years) & $21.7 \pm 1.5$ & $21.98 \pm 3.6$ & 0.12 \\
\hline BMI & $19.5 \pm 2.3$ & $19.6 \pm 2.1$ & 0.42 \\
\hline WHR & $0.69 \pm 0.12$ & $0.75 \pm 0.78$ & 0.31 \\
\hline Psychological stress (PSS) & & & 0.28 \\
\hline Family stress & $12.75 \pm 2.3$ & $18.2 \pm 1.6$ & $19.6 \pm 2.3$ \\
\hline Study stress & $13.8 \pm 1.8$ & $21.45 \pm 3.4$ & $16.8 \pm 2.8$ \\
\hline Economic stress & $10.7 \pm 2.4$ & $56.4 \pm 4.6$ & \\
\hline Social stress & $9.95 \pm 3.1$ & $28.8 \pm 5.8$ & \\
\hline Total PS score & & & \\
\hline
\end{tabular}

Values are expressed as mean $\pm \mathrm{SD}$; the unpaired $\mathrm{t}$ - test was used for within and between groups. Values at $\mathrm{p}<0.05$ were considered statistically significant; BMI: body mass index; WHR: waist to hip ratio. PSS: Psychological stress score

Table 2: Psychological stress scores (PSS) in studied Students of Faculty of Sport Sciences Pr- and Post- of PMR interventions training program for 12 weeks (means $\pm S D ; n=40$ ), Values are expressed as mean $\pm S D ; *$ P $<0.01,{ }^{*} P<0.001$. Significance at $\mathrm{p}<0.05$, PMR: Progressive muscle relaxation; PSS: Psychological stress score

\begin{tabular}{|c|c|c|c|c|}
\hline Parameters & Control Group (n=20) & & Psychology stressGroup (n=20) & Post \\
\hline & Pre & Post & Pre & $12.6 \pm 1.5^{*}$ \\
\hline Family stress & $12.75 \pm 2.3$ & $11.5 \pm 2.5^{*}$ & $18.2 \pm 1.6$ & $13.45 \pm 1.9^{*}$ \\
\hline Study stress & $13.8 \pm 1.8$ & $9.3 \pm 1.68^{*}$ & $19.6 \pm 2.3$ & $9.8 \pm 1.91^{*}$ \\
\hline Economic stress & $10.7 \pm 2.4$ & $7.2 \pm 1.9^{*}$ & $21.45 \pm 3.4$ & $8.75 \pm 2.3^{*}$ \\
\hline Social stress & $9.95 \pm 3.1$ & $8.5 \pm 1.52^{*}$ & $16.8 \pm 2.8$ & $44.6 \pm 3.8^{*}$ \\
\hline Total PS score & $28.8 \pm 5.8$ & $22.5 \pm 3.5^{*}$ & $56.4 \pm 4.6$ & \\
\hline
\end{tabular}

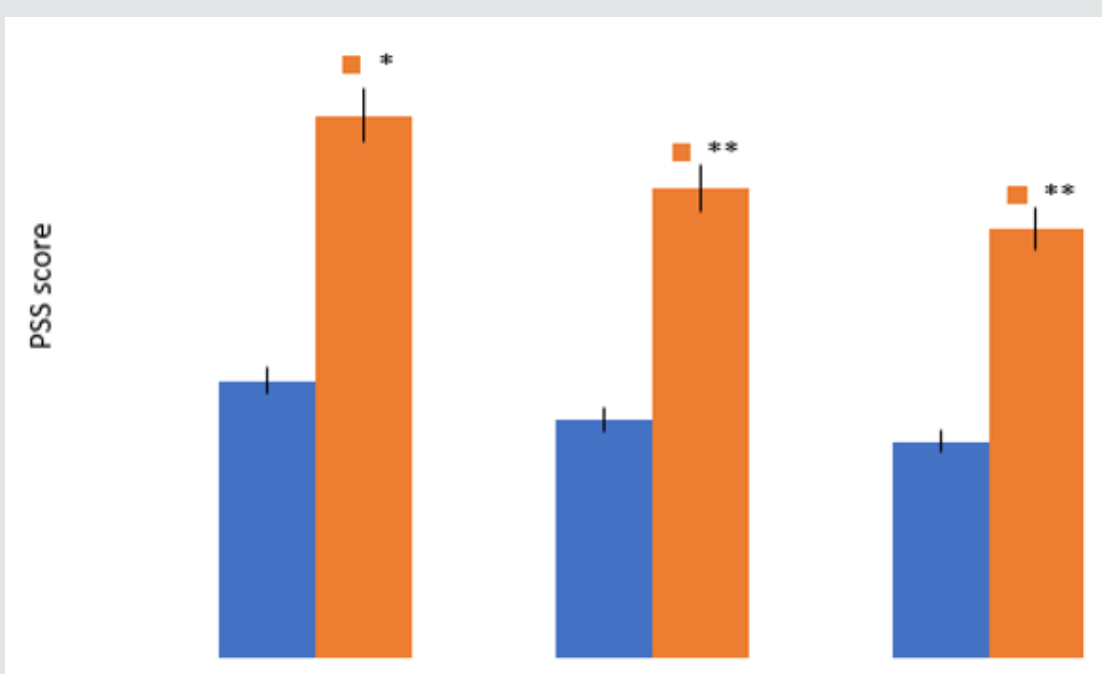

- Control

Psy. stress

Figure 1: Mean Psychological stress (PSS )scores at three time points [Pre, during (D) and post-Progressive muscle relaxation training interventions of participants (mean SD)]. Between groups: ${ }^{*}<0.05 ;{ }^{* *} p<0.001$. PMR: Progressive muscle relaxation; PSS: Psychological stress score; 


\section{Discussion}

In this study, psychological stress paramterswere significantly reported in $50 \%$ ( 20/40) of the participated students. These results greatly require to find out more non-drug supportive interventions for the reduction of the psychological stress among younger ages. The data matched with those who reported significant collateral damage associatwed with severe diseases among both older and younger ages [1,2]. A harmful consequences were reported among youth and young students [3-5], in which disturbances were reported in all psychological, physiological and behavioral proceed [2]. In addition, psychological stress was reported as a major risk factor for the development and progression of a number of diseases, including cardiovascular disease, cancer, arthritis, and major depression [1]. These consequences in turn greatly affects in many emotional, behavioral, sexual, economic, academic and social conflicts of young ages and result in social and physical changes [6,7]. Recently, the importance of psychological skills training (PST) techniques and other relating training techniques have been recognized, and the number of studies on athletes or other patient subjects using psychological training strategies to improve psychological status [19-21]. One of the most training techniques affecting on psychological stress is Progressive muscle relaxation (PMR) [9-11]. It showed to reduce a stress and anxiety by alternately tensing and relaxing the muscles as previously reported [9-11]. It mainly requires both a physical and a mental component of the subject during treatment sessions. In this current study, both control and psychological students participated in PMR training interventions for 12 weeks (2 times /week). PMR-Post results showed that all psychological parameters were significantly declined and improved in all healthy ( $\mathrm{P}=0.01)$ and students with psychological stress $(\mathrm{P}=0.001)$. The data showed that PMR training interventions program improve psychological stress gradually during the period of treatment which support its efficacy as nondrug strategy for treating psychological stress and its consequences among younger ages.

Previous research reports recommended several benefits of PMR technique including a reduction in salivary cortisol levels and generalized anxiety [10], decreased blood pressure and heart rate $[10,12]$, decreased headaches [13], better management of cardiac rehabilitation [14] improvement of quality of life of patients with chronic diseases or following severe surgical operations [15-16]. Thus it is better to use PMR technique in for ameliorating psychological stress among younger ages. Previous treatment strategies with predominant skeletal muscle components aslotend to produce greater muscular effects particularly; a decrease in muscle tone and tension. Different versions including, PMR, biofeedback, yoga, and systematic breathing are the most investigated techniques in this domain [22]. Progressive muscle relaxation and its derivations have received the most attention in scientific context whereas it significantly deliberate and continuously reduce of tension in specific muscle groups of the locomotor system [9]. A review on the use of PMR in clinical trials revealed moderate to large effect sizes on the effectiveness of PMR $[23,24]$.

\section{Conclusion}

In this study, PMR training interventions for 12 weeks significantly improve psychological stress among the students of faculty of sports sciences. The data showed that PMR training interventions program improve psychological stress gradually during the period of treatment which supports the use of this nondrug treatment among psychological stress holders, particularly in younger ages.

\section{Acknowledgement}

Support for this work was provided by the Deanship of Scientific Research, and the College of Sports Sciences and Physical Activity Research Center, king Saud university, Riyadh, Saudi Arabia

\section{References}

1. Muscatell KA, Eisenberger NI (2012) A social neuroscience perspective on stress and health. Soc Personal Psychology Compass 6(12): 890-904

2. Cooper CL, Dewe PJ, O'Driscoll MP (2003) Organizational stress. A review and critique of theory, research, and applications. SAGE publications 10(1): 130-131.

3. Dweck CS (1990) Self theories: their role in motivation, personality and development. Nebr Symp Motivation 38: 199-235

4. Ford IW, Eklund RC, Gordon S (2000) An examination of psychosocial variables moderating the relationship between life stress and injury time loss among athletes of a high standard. Journal of Sports Science 18(5): 301-312

5. Hayama Y, Inoue T (2012) The effects of deep breathing on tensione anxiety and fatigue in cancer patients undergoing adjuvant chemotherapy. Complement Therapy Clinical Practice 18(2): 94-98.

6. Kaya M, Genç M, Kaya B, Pehlivan E (2007) Prevalence of depressive symptoms, ways of coping, and related factors among medical school and health services higher education students. Turkish Journal of Psychiatry 18(1): 137-146.

7. Kerimova M (2000) Examination of psychological symptoms seen in high school students according to some variables. Unpublished master dissertation Ankara.

8. Melnyk BM, Jacobson D, Kelly S, Haver OJ, Small L, et al. (2009) Improving the mental health, healthy lifestyle choices, and physical health of Hispanic adolescents: A randomized controlled pilot study. Journal of School Health 79(12): 575-584

9. Jacobson E (1938) Progressive relaxation. Chicago: University of Chicago Press.

10. Pawlow LA, Jones GE (2002) The impact of abbreviated progressive muscle relaxation on salivary cortisol. Biological Psychology 60(1): 1-16.

11. Titlebaum HM (1998) Relaxation. Holistic Nursing Practice 2(3): 17-25.

12. Sheu S, Irvin BL, Lin HS, Mar CL (2003) Effects of Progressive Muscle Relaxation on Blood Pressure and Psychosocial Status for Clients with Essential Hypertension in Taiwan. Holistic Nursing Practice 17(1): 4147.

13. Anderson RE, Seniscal CA (2001) Comparison of selected osteopathic treatment and relaxation for tension-type headaches. Headache 46(8): 1273-1280.

14. Wilk C, Turkoski B (2001) Progressive muscle relaxation in cardiac rehabilitation: a pilot study. Rehabilitation Nursing 26(6): 238-242.

15. Dehdari T, Heidarnia A, Ramezankhani A, Sadeghian S, Ghofranipour F (2009) Effects of progressive muscular relaxation training on quality of 
life in anxious patients after coronary artery bypass graft surgery. Indian Journal of Medical Research 129(5): 603-608.

16. Ghafari S, Ahmadi F, Nabavi M, Anoshirvan K, Memarian R, et al. (2009) Effectiveness of applying progressive muscle relaxation technique on quality of life of patients with multiple sclerosis. Journal Clinical Nursery 18(15): 2171-2179.

17. Bilgel N, Bayram N (2010) Turkish Version of the Depression Anxiety Stress Scale (DASS-42): Psychometric Properties. Archives of Neuropsychiatry 47(2): 118-126.

18. Lovibond PF, Lovibond SH (1995) The structure of negative emotional states: Comparison of the Depression Anxiety Stress Scales (DASS) with the Beck Depression and Anxiety Inventories. Behaviour Research and Therapy 33: 335-343

19. Mujica I (2009) Intense training: the key to optimal performance during the taper. Paper for the International Consensus Conference Performance in Top Sports Involving Intense Exercise Copenhagen 2: 24-31
20. Weinberg RS, Gould D (2007) Foundations of sport and exercise psychology. ( $4^{\text {th }}$ edn.), Human Kinetics.

21. Whitmarsh BG, Alderman RB (1993) Role of psychological skills training in increasing athletic pain tolerance. Sports Psychology 7(4): 388-399.

22. Kudlakova K, Eccles DW, Dieffenbach K (2013) Use of relaxation skills in differentially skilled athletes. Psychology of Sport and Exercise 14(4): 468-475.

23. Dolbier CL, Rush TE (2012) Efficacy of abbreviated progressive muscle relaxation in a high-stress college sample. International Journal of Stress Management 19(1): 48-68.

24. Crawford C, Wallerstedt DB, Khorsan R, Clausen SS, Jonas WB, et al. (2013) A systematic review of biopsychological training programs for the self management of emotional stress: Potential applications for the military. Evidence-basedComplementary and Alternative Medicine.

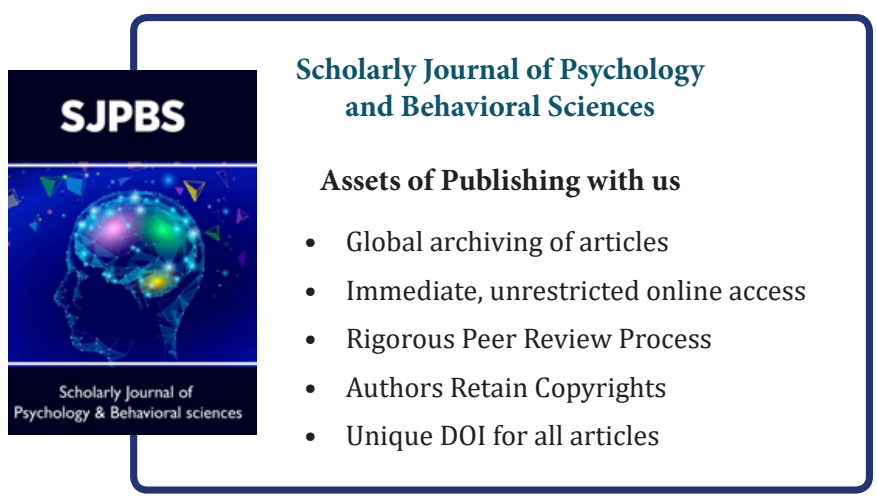

